\title{
Radiation treatment planning for prostate cancer: A new dosimetric comparison of five and seven fields IMRT plans
}

\section{S.R.M. Mahdavi', E. Jazayeri Gharehbagh', A.R. Nikoofar ${ }^{2 *}$, B. Mofid3, M. Vasheghani 4 , D. Saedi5}

\author{
${ }^{1}$ Department of Medical Physics, Iran University of Medical Sciences, Tehran, Iran \\ ${ }^{2}$ Department of Radiation Oncology, Iran University of Medical Sciences, Tehran, Iran \\ ${ }^{3}$ Department of Radiation Oncology, Shahid Beheshti university of Medical Sciences, Tehran, Iran \\ ${ }^{4}$ Department of Radiotherapy, Pars General Hospital, Keshavarz Bolv., Tehran Iran \\ ${ }^{5}$ Department of Radiology, Pars General Hospital, Keshavarz Bolv., Tehran Iran
}

\section{- Original article}

*Corresponding author:

Dr. Ali Reza Nikoofar,

Fax: + 982188985887

E-mail:

arnikoofar@gmail.com

Revised: July 2015

Accepted: Aug. 2015

Int. J. Radiat. Res., April 2017; 15(2): 177-183

DOI: 10.18869 /acadpub.ijrr.15.2.177

\begin{abstract}
Background: To compare the dosimetric coverage of the planning target volume (PTV) and the dose delivered to the main Organs at Risk (OARs) in 5 and 7-field techniques of Intensity Modulated Radiation Therapy (IMRT) in patients with local prostate cancer. Materials and Methods: Twelve patients with local prostate cancer underwent 5 and 7 -field IMRT planning. The delivery of IMRT was carried out using the sliding technique. The dose coverage for PTV was designated to $\geq 98 \%$ of the PTV covered by $95 \%$ of the prescribed dose. Dose conformity was evaluated by comparing the volume of nontarget tissue receiving maximum, and average of the prescribed dose and the dose of $33 \%, 50 \%$, and $66 \%$ of the volumes on both planning sets. For target, this evaluation was made with comparing the Conformity Index (Cl) and Inhomogeneity Index (HI). In addition, we compared the monitor units used for dose delivery in both planning techniques. Results: All the 5 and 7field IMRT plans differed slightly in the measured parameters, and none of them have statistically significant differences with each other except for the monitor units where significant differences were observed in favor of the 5field IMRT plans $(p=0.000)$. In all of the 5 -field IMRT plans the mean dose delivered to OARs were very similar or less than that of the 7-field plans. Conclusion: In comparison to the 7-field technique, the 5-field IMRT technique has resulted in improved IMRT dose conformity, homogeneity, and lesser MUs used for radiation therapy. However, this difference was not significant.
\end{abstract}

Keywords: IMRT, dose conformity, MUs, prostate cancer

\section{INTRODUCTION}

Intensity-modulated Radiotherapy (IMRT) is increasingly being used worldwide for a variety of cancers and rapidly becoming part of mainstream radiation oncology. It achieves better conformity of radiation dose to the target and normal structures and offers reduced critical structure dose compared to the conventional Three-dimensional Conformal
Radiotherapy (3D-CRT) approaches(1).

The basic principle of IMRT involves irradiation from a number of different directions with beams of nonuniform energy fluences, which have to be optimized for delivering a high dose to the target volume and acceptable low dose to the surrounding normal structures such as the rectum and the penile bulb in prostate radiotherapy (2). However, one of the most significant obstacles for the implementation of 
IMRT has been slower throughput because of longer treatment delivery times. The problem is compounded by the requirement for more stringent setup techniques, and for some site imaging for target localization before treatment and more Monitor Units (MUs) used for treatment ${ }^{(3)}$.

Treatment accuracy has been improved with Image-guided Radiotherapy (IGRT) $(4 ; 5)$. IMRT and IGRT are complementary technologies. On this base, the American College of Radiology has recently deemed IMRT to be the most appropriate treatment planning and treatment delivery approach for prostate cancer(6).

IMRT of prostate uses five to seven beams for treatment. Treatment planning program divides each beam into a large number of beamlets and determines the optimum setting of their energy fluences or beam weights. IMRT increases the volume of normal tissue exposed to some radiation but can reduce the total dose received by critical organs (7), permit tumor dose escalation, thereby yielding higher rates of local tumor control (8).

The aim of this study was to compare the dose to normal tissues and dose-limiting structures such as the rectum, dose homogeneity and conformity and the number of MUs required for delivery, when using the same prescription doses, planning system and PTV margins with five and seven beam IMRT plans.

\section{MATERIALS AND METHODS}

Prior to initiating the study, a university ethics approval consent was obtained from twelve patients previously treated for prostate cancer with IMRT, and comparisons were made between the original and new plans. Treatment plans were generated using a five beams arrangement with gantry angles of $15^{\circ}, 55^{\circ}$, $110^{\circ}, 260^{\circ}, 330^{\circ}$ and seven beams arrangement with gantry angles of $0^{\circ}, 52^{\circ}, 103^{\circ}, 154^{\circ}, 205^{\circ}$, $256^{\circ}, 308^{\circ}$ and dynamic multi leaf collimator (MLC) delivery for each patient. Planning method and comparisons were made between two plans for each patient. Target coverage and normal tissue constraints were those designated Int. J. Radiat. Res., Vol. 15 No. 2, April 2017 by RTOG protocols and were kept consistent among all the plans ${ }^{(3,9)}$. Dose conformity was evaluated through Dose-Volume Histogram (DVH) analysis for both target volume and surrounding normal structures.

All patients had histologically confirmed and clinically staged localized prostate cancer. The age of the patients ranged from 57 to 80 years (the mean being 71.6 years). Initial prostate-specific antigen was in the range of $5.9-16.4 \mathrm{ng} / \mathrm{ml}$ (the mean being $11.3 \mathrm{ng} / \mathrm{ml}$ ) with Gleason scores of 6 to 8 . All patients received neoadjuvant hormonal therapy.

Treatment plans were produced and analyzed using the TIGRT, treatment planning system (Linatec Company....) with doses calculated over an isotropic dose grid with 3, 3 and $5 \mathrm{~mm}$ spacing for Clinical Tumor Volume (CTV), Planning Tumor Volume (PTV) and Organs at Risk (OARs), respectively using a collapsed-cone convolution algorithm. Plans were produced for treatment on Primus Series Oncology Systems (Siemens Company....), linear accelerators at beam energy of $15 \mathrm{MV}$. In all cases, the field shape of treatment plans were made using external multi leaf collimators with a leaf width of 0.5 to $1 \mathrm{~cm}$ at the isocentre from the center to the edge of the field and according to each of the two trial protocols.

Patients were scanned in the treatment position from the level of L5-S1 to the ischial tuberosities. They were placed in a supine position and asked to keep their rectum empty and bladder comfortably full at the time of simulation and during each treatment fraction. No immobilization was used. The planning CT scans were taken with a $5 \mathrm{~mm}$ thickness. To produce Digitally Reconstructed Radiographs (DRR), CT slices were reconstructed at $2 \mathrm{~mm}$ increments throughout the scanned volume. The volumetric CT data set was transferred to the planning system. The PTV, bladder, rectum and femora were contoured. The PTV included the entire prostate and seminal vesicles plus a $5 \mathrm{~mm}$ margin, except at the prostate-rectum interface where a $3 \mathrm{~mm}$ margin was used to decrease the risk of rectal toxicity (2). On-line portal imaging was used to decrease treatment uncertainties and assure the quality of treatment delivery. 
Then, the DDR of each field was generated. All of the plans were evaluated with dose of 80Gy in 40 fractions.

Delivery of IMRT was carried out using the sliding window technique as developed by Spirou and Chui (10). The desired beam intensity profiles were delivered by DMLC (11).

The definitions of CTV and PTV for our trial and for both low to moderate risk patients are shown in table $1(2)$.

The aim of these planning methods was to obtain dosimetric coverage of the nominated PTV as designated by the protocol and its dose constraints while evaluating the dose delivered to the main OARs, which are the rectum and bladder. Dose volume histograms were produced for PTVs and all pertinent OARs, to allow objective and quantitative comparison of the dose distributions between the two different IMRT planning techniques. Figure 1 shows two IMRT plans.

The dose coverage for both PTV1 and PTV2 was designated to an International Commission Radiation Units and Measurements report 62 reference point in the PTV, with $\geq 98 \%$ of the PTV covered by $95 \%$ of the prescribed dose. The goal was to keep the maximal point dose at $\leq 107 \%$.

Dose conformity was evaluated by comparing the volume of non-target tissue receiving maximum, minimum, and average of the prescribed dose and the dose delivered to 33\%, $50 \%$, and $66 \%$ of the volumes (Dmax, Dmin, Dmean, D33\%, D50\%, and D66\%) on both planning sets. For target, this evaluation was made with comparing the Conformity Index (CI) and Inhomogeneity Index (HI).

1. Dmax: the absolute maximum dose received by any point in the OARs or PTV (in Gy)

2. Dmin: the absolute minimum dose received by any point in the OARs or PTV (in Gy)

3. Dmean: the absolute mean dose received by the OARs or PTV (in Gy)

4. D33\%: the absolute dose received by the 33\% of the OARs volume (in Gy)

5. D50\%: the absolute dose received by the $50 \%$ of the OARs volume (in Gy)

6. D66\%: the absolute dose received by the $66 \%$ of the OARs volume (in Gy)

7. D $2 \%$ : the absolute dose received by the $2 \%$ of the PTV volume (in Gy)

Table 1. Definition of target volumes.

\begin{tabular}{|c|c|c|}
\hline & Low risk & Moderate risk \\
\hline CTV1 & Prostate + base of seminal vesicles & Prostate + seminal vesicles \\
\hline PTV1 & CTV1+5mm* & CTV1+5mm* \\
\hline CTV2 & Prostate & Prostate \\
\hline PTV2 & Prostate & Prostate \\
\hline
\end{tabular}

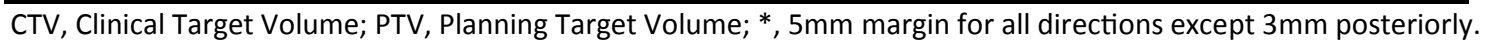

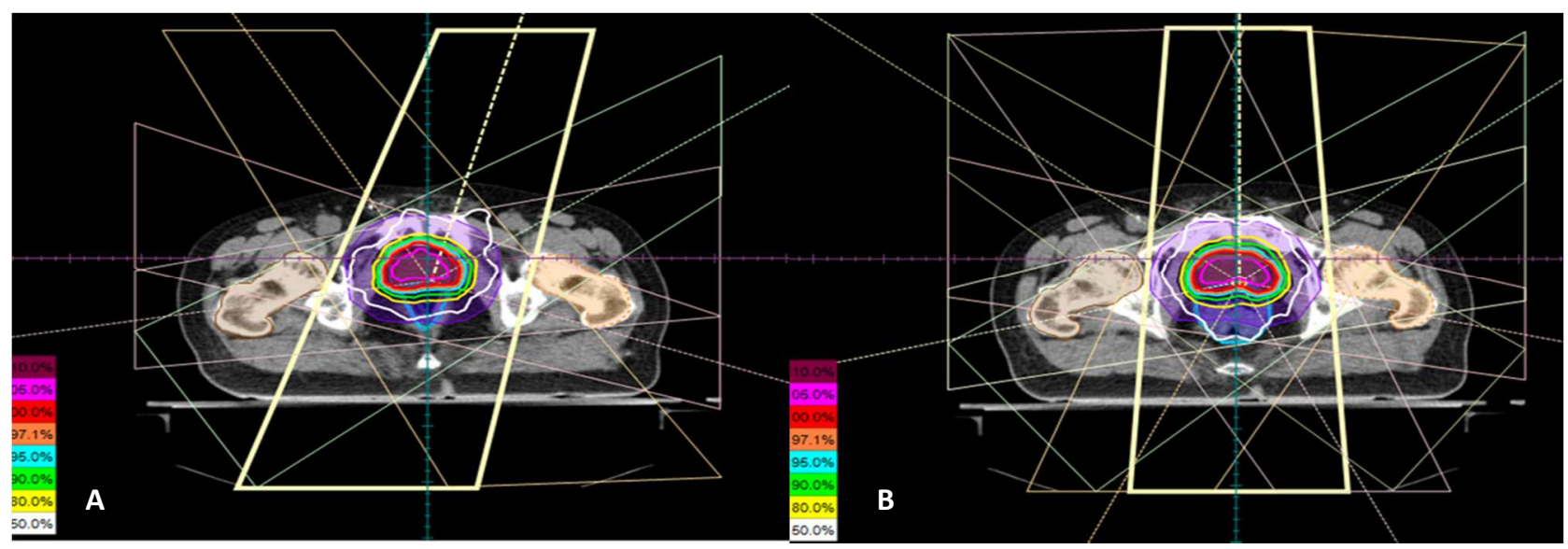

Figure 1. Axial displays of treatment planning's show irradiation beams and dose distribution in the target and overlying structures: a; 5-field and, b; 7-field IMRT plans. 
8. D98\%: the absolute dose received by the $98 \%$ of the PTV volume (in Gy)

For normal tissues, we compared the doses treating $33 \%, 50 \%$, and $66 \%$ of the organ volumes in two IMRT plans (12). We also looked at the maximum doses, as well as volumes of organs that received $102 \%$ of the treating dose, to achieve a more representative comparison of DVHs between 5 and 7-fields IMRT plans.

The difference between the two planning techniques was compared using the mean statistics for their radiated OARs volumes and PTV coverage as the main parameter. An independent Student's t-test was used to verify the significance of the differences in the mean outcome of the treatment plans. The data was normally distributed and a $P$ value of $\leq 0.05$ was taken into account as the significant difference.

\section{RESULTS}

All the 5 and 7-field IMRT plans differed slightly in the measured parameters, however none of them have statistically significant differences with each other except for the monitor units where significant differences were observed in favor of the 5-field IMRT plans $(p=0.000)$.

Table 2. D33\%, D50\%, and D66\% for rectum in Two IMRT plans.

\begin{tabular}{|c|c|c|c|c|c|c|}
\hline \multirow{3}{*}{ Patient } & \multicolumn{6}{|c|}{ Rectum } \\
\hline & \multicolumn{2}{|c|}{ D33\% (Gy) } & \multicolumn{2}{|c|}{ D50\%(Gy) } & \multicolumn{2}{|c|}{ D66\%(Gy) } \\
\hline & 5-field & 7-field & 5-field & 7-field & 5-field & 7field \\
\hline$\overline{1}$ & 65.24 & 64.84 & 52.00 & 48.16 & 36.41 & 50.22 \\
\hline 2 & 59.14 & 59.14 & 47.73 & 50.85 & 36.94 & 46.58 \\
\hline 3 & 63.44 & 65.52 & 52.81 & 55.52 & 44.23 & 50.53 \\
\hline 4 & 60.26 & 58.58 & 46.17 & 51.03 & 36.22 & 44.85 \\
\hline 5 & 59.25 & 60.30 & 49.90 & 53.17 & 49.18 & 42.26 \\
\hline 6 & 63.23 & 61.09 & 50.25 & 51.96 & 41.52 & 46.87 \\
\hline 7 & 69.61 & 61.62 & 54.87 & 51.58 & 40.96 & 45.54 \\
\hline 8 & 53.14 & 57.38 & 42.99 & 51.19 & 34.40 & 44.47 \\
\hline 9 & 54.06 & 55.51 & 40.50 & 46.74 & 28.10 & 27.93 \\
\hline 10 & 60.42 & 57.49 & 43.34 & 51.03 & 32.14 & 40.82 \\
\hline 11 & 57.49 & 54.78 & 42.62 & 47.82 & 33.45 & 35.29 \\
\hline 12 & 53.42 & 54.67 & 41.09 & 45.09 & 9.36 & 9.36 \\
\hline Mean & $5.00 \pm 59.89$ & $3.60 \pm 59.24$ & $4.92 \pm 47.02$ & $2.90 \pm 50.34$ & $9.94 \pm 35.24$ & $11.64 \pm 40.39$ \\
\hline
\end{tabular}

The mean, maximum and minimum doses for PTVs in 5-field and 7- field IMRT plans were in the same ranges 81.63, 86.78 and 68.51 Gy and 81.71, 87.61 and 68.76 Gy respectively (Table 3$)$. Statistically, there were no significant differences between the two IMRT plans ( $P=0.882,0.341$, and 0.919 respectively).

Int. J. Radiat. Res., Vol. 15 No. 2, April 2017
The delivered doses to $33 \%, 50 \%$, and $66 \%$ of the rectum volumes were 59.89, 47.02 and, 35.24 Gy for 5-field and 59.24, 50.34, and 40.39 Gy for 7-field IMRT plans respectively (Table 2). As we can see the dose of $50 \%$ and $66 \%$ of the rectum volumes in 5-field plan are less than that of 7-field plans. However, statistically, there were no significant differences between them $(p$ values $=0.059$, and 0.256 , respectively) .

Maximum doses delivered to the rectum in both of IMRT plans were in comparable range from 86.78 to 87.62 Gy ( $p=0.234$ ) for 5 and 7 -fields respectively. The measured doses of $2 \mathrm{~cm}^{3}$ of the rectum volumes were similar in both plans $1.00 \%$ and $1.01 \%$ of the total prescribed dose respectively with $p=0.379$ ).

Table 4 shows the doses which were delivered to the bladder volumes in two plans. Bladder statistics revealed no significant differences between the doses delivered to the $33 \%, 50 \%$ and $66 \%$ of the organ volumes $(P=0.856, p=0.955$, and $p=0.145$ respectively). Maximum doses delivered to the bladder were similar 84.47 and $84.71 \mathrm{~Gy}$ for 5 and 7-field IMRT plans respectively $(p=0.803)$. Although, the average dose delivered to the bladder with 5field IMRT plan were less than that delivered by 7 -field IMRT plan, but there were not significant differences between them ( $p=0.703)$. 
Table 3. Maximum, Minimum, and Mean target dose for PTVs in 5 and 7-field IMRT plans.

\begin{tabular}{|c|c|c|c|c|c|c|}
\hline \multirow{2}{*}{ patient } & \multicolumn{3}{|c|}{ IMRT 5- field (PTV) } & \multicolumn{3}{c|}{ IMRT 7- field (PTV) } \\
\cline { 2 - 7 } & Dmax(Gy) & Dmin(Gy) & Dmean(Gy) & Dmax(Gy) & Dmin(Gy) & Dmean(Gy) \\
\hline $\mathbf{1}$ & 88.83 & 63.04 & 82.59 & 88.27 & 71.03 & 83.21 \\
\hline $\mathbf{2}$ & 84.82 & 77.33 & 79.72 & 84.92 & 77.47 & 79.68 \\
\hline $\mathbf{3}$ & 84.44 & 57.57 & 79.99 & 84.44 & 57.57 & 79.99 \\
\hline $\mathbf{4}$ & 86.91 & 67.05 & 81.95 & 88.33 & 63.97 & 82.05 \\
\hline $\mathbf{5}$ & 86.32 & 74.22 & 81.89 & 88.38 & 74.25 & 82.09 \\
\hline $\mathbf{6}$ & 85.56 & 60.43 & 81.71 & 85.78 & 62.38 & 81.70 \\
\hline $\mathbf{7}$ & 87.14 & 65.60 & 79.22 & 87.60 & 67.41 & 79.46 \\
\hline $\mathbf{8}$ & 85.27 & 74.89 & 81.76 & 84.91 & 67.66 & 81.82 \\
\hline $\mathbf{9}$ & 87.29 & 72.36 & 82.29 & 93.30 & 69.75 & 82.65 \\
\hline $\mathbf{1 0}$ & 88.51 & 68.72 & 82.12 & 89.77 & 66.51 & 82.42 \\
\hline $\mathbf{1 1}$ & 88.99 & 68.54 & 82.93 & 89.30 & 72.92 & 82.84 \\
\hline $\mathbf{1 2}$ & 87.29 & 72.36 & 83.41 & 86.39 & 74.14 & 82.53 \\
\hline mean & $\mathbf{1 . 5 4 \pm 8 6 . 7 8}$ & $\mathbf{6 . 0 8} \pm \mathbf{6 8 . 5 1}$ & $\mathbf{1 . 3 1} \pm \mathbf{8 1 . 6 2}$ & $\mathbf{2 . 5 4 \pm 8 7 . 6 1}$ & $\mathbf{5 . 6 8} \pm \mathbf{6 8 . 7 6}$ & $\mathbf{1 . 2 8} \pm \mathbf{8 1 . 7 1}$ \\
\hline
\end{tabular}

PTV: Planning Target Volume

Table 4. D33\%, D50\%, and D66\% for bladder in two IMRT plans.

\begin{tabular}{|c|c|c|c|c|c|c|}
\hline \multirow{3}{*}{ Patient } & \multicolumn{6}{|c|}{ Bladder } \\
\hline & \multicolumn{2}{|c|}{ D33\%(Gy) } & \multicolumn{2}{|c|}{ D50\%(Gy) } & \multicolumn{2}{|c|}{ D66\%(Gy) } \\
\hline & 5-field & 7-field & 5-field & 7-field & 5-field & 7-field \\
\hline 1 & 56.54 & 56.18 & 47.32 & 48.16 & 41.41 & 41.86 \\
\hline 2 & 48.18 & 48.37 & 42.34 & 37.22 & 26.90 & 29.14 \\
\hline 3 & 51.51 & 53.06 & 39.69 & 39.96 & 2.40 & 31.48 \\
\hline 4 & 45.13 & 44.69 & 38.33 & 34.56 & 25.87 & 26.53 \\
\hline 5 & 50.00 & 53.03 & 41.82 & 42.63 & 34.60 & 33.98 \\
\hline 6 & 41.22 & 39.21 & 23.43 & 27.20 & 10.67 & 18.15 \\
\hline 7 & 52.74 & 50.53 & 41.89 & 38.38 & 24.39 & 30.75 \\
\hline 8 & 40.81 & 36.33 & 24.75 & 25.79 & 18.14 & 18.28 \\
\hline 9 & 61.06 & 61.64 & 47.50 & 49.42 & 34.18 & 35.53 \\
\hline 10 & 52.74 & 53.70 & 44.43 & 42.14 & 35.16 & 35.16 \\
\hline 11 & 33.58 & 39.67 & 14.11 & 19.71 & 0.71 & 1.06 \\
\hline 12 & 61.15 & 59.99 & 48.24 & 48.05 & 39.38 & 37.61 \\
\hline mean & $8.33 \pm 49.55$ & $8.23 \pm 49.70$ & $11.01 \pm 37.80$ & $9.48 \pm 37.77$ & $13.85 \pm 24.49$ & $11.15 \pm 28.30$ \\
\hline
\end{tabular}

The volumes of rectum and bladder which have received $102 \%$ of the total dose were very similar, specifically 2.30 and $2.32 \mathrm{~cm}^{3}$ for the rectum and 2.52 and $2.46 \mathrm{~cm}^{3}$ for the bladder, with $p=0.976$, and $p=0.921$ respectively. In addition, the doses that have received by $2 \mathrm{~cm}^{3}$ of the rectum volumes have not exceeded from $100.1 \%$ except in one patient who received $103 \%$ of prescribed dose for that.

Compared to the 7-field IMRT plans, the 5field plans achieved a $5.7 \%$ relative decrease in the mean number of MUs required for radiation delivery (615 MU vs. 558 MU respectively) as shown in table 5.

Table 5. Comparison of the mean dosimetric parameters.

\begin{tabular}{|c|c|c|c|}
\hline variable & Mean & Mean & $\begin{array}{c}\text { Significant at } \\
\text { p } \leq 0.05\end{array}$ \\
\hline Monitor units & $29.29 \pm 558$ & $26.48 \pm 615$ & $* 0.000$ \\
\hline PTV volume of 95\% isodose (cm ${ }^{3}$ ) & $54.45 \pm 221.6$ & $56.8 \pm 224.77$ & 0.890 \\
\hline Conformity index (volume of 95\%/PTV)(13) & $0.27 \pm 1.589$ & $0.26 \pm 1.568$ & 0.844 \\
\hline Inhomogeneity index (D2\%-D98\%)/D mean & $0.04 \pm 0.03$ & $0.01 \pm 0.02$ & 0.285 \\
\hline
\end{tabular}

PTV; Planning Target Volume, SD; Standard Deviations 


\section{DISCUSSION}

The outcome of treatment for prostate cancer patients, measured as PSA progression-free survival, has improved in recent years. The improvement was mainly due to the radiation dose escalation (14) and addition of the androgen suppression therapy (15).

Safe increase in the radiation dose to the prostate gland is limited by the radiation toxicity effects on normal tissues, particularly the rectum(16).The major advantage of IMRT is the ability to decrease the dose to critical structures, which in turn lowers the radiation toxicity effects $(17,18)$.

The increase of normal tissue integral dose with multiple beam radiation therapy during IMRT is also a major concern due to the potential risk of the secondary induced malignancies especially in younger patients (7). The large number of beamlets and monitor units can lead to an increase in integral dose $(7,19)$, however, a higher-energy photons beams may reduce the normal tissue integral doses (20).

Long time follow-up is needed for the detection of secondary malignancies after radiation therapy and because IMRT is a relatively new technique, the true risk of second malignancies is not yet known (7). However, even in the absence of such data, the theoretical increased risk of second malignancies is sufficient explanation to attempt to reduce the MUs delivered to patients while maintaining a high dose to the target volumes (21).

In our study, 7-field plans use a greater number of MUs per treatment than 5-field IMRT plans which lead to greater interleaf leakage dose and therefore, again lead to an increasing rate of probability of cancer induction (7). An increase in MUs also leads to the greater linear accelerator beam-on time and consequently more treatment time and running cost. However, it should be mentioned that more MUs in IMRT techniques results into a more or less better homogeneity of dose distribution within the PTV and it is obvious in 7-field IMRT technique (table 5) which requires a clinical judgment.

From the other points of view using high energy beam with a larger MUs may lead to increase in neutron production during photon-neutron interaction, which its potential risk must also be weighed against perceived benefits (22).

\section{CONCLUSION}

The Intensity Modulated Radiotherapy (IMRT) is a novel form of radiation delivery technique in our country (Iran). The 5-field fixed gantry, sliding window IMRT is the standard IMRT configuration used to treat patients at our center (Pars hospital radiotherapy department). However, in comparison with the 7-field technique, the 5-field IMRT technique, in addition to dose homogeneity, has resulted in improved IMRT dose conformity, and lesser MUs for patient irradiation. In fact, except for MUs, the differences between these two techniques are very small and not statistically significant, however, their own clinical outcomes needs a long time study and more investigation.

\section{Conflicts of interest: Declared none.}

\section{REFERENCES}

1. Vaarkamp J, Malde R, Dixit S, Hamilton C (2009) A comparison of conformal and intensity modulated treatment planning techniques for early prostate cancer. Journal of Medical Imaging and Radiation Oncology, 53: 310-7.

2. South C, Khoo V, Naismith O, Norman A, Dearnaley D (2008) A comparison of treatment planning techniques used in two randomised UK external beam radiotherapy trials for localised prostate cancer. Clinical Oncology, 20 (1): 15-21.

3. Price Jr RA, Murphy S, McNeeley SW, Ma C-MC, Horwitz E, et al. (2003) A method for increased dose conformity and segment reduction for SMLC delivered IMRT treatment of the prostate. International Journal of Radiation Oncology* Biology* Physics, 57: 843-52.

4. Martin JM, Bayley A, Bristow R, Chung P, Gospodarowicz $M$, et al. (2009) Image guided dose escalated prostate radiotherapy: still room to improve. Radiat Oncol, 4(50): 717.

5. Kupelian PA, Willoughby TR, Reddy CA, Klein EA, Mahadevan A (2008) Impact of image guidance on outcomes after external beam radiotherapy for localized prostate cancer. International Journal of Radiation Oncology* Biology* Physics, 70(4): 1146-50. 
6. Michalski JM, Roach III M, Merrick G, Anscher MS, Beyer DC, et al. (2009) ACR Appropriateness Criteria ${ }^{\circledR}$ on External Beam Radiation Therapy Treatment Planning for Clinically Localized Prostate Cancer: Expert Panel on Radiation Oncology-Prostate. International Journal of Radiation Oncology* Biology* Physics, 74(3): 667-672.

7. Hall EJ and Wuu CS (2003) Radiation-induced second cancers: the impact of 3D-CRT and IMRT. International Journal of Radiation Oncology* Biology* Physics, 56(1): 83-88.

8. Zelefsky MJ, Fuks Z, Happersett L, Lee HJ, Ling CC, et al. (2000) Clinical experience with intensity modulated radiation therapy (IMRT) in prostate cancer. Radiotherapy and Oncology, 55(3): 241-249.

9. Yan D, Vicini F, Wong J, Martinez A (1997) Aadaptive radiation therapy. Physics in medicine and Miology, 42(1): 123.

10. Spirou SV and Chui CS (1994) Generation of arbitrary intensity profiles by dynamic jaws or multileaf collimators. Medical physics, 21(7): p. 1031-1041.

11. Ling CC, Burman C, Chui CS, Kutcher GJ, Leibel SA, et al. (1996) Conformal radiation treatment of prostate cancer using inversely-planned intensity-modulated photon beams produced with dynamic multileaf collimation. International Journal of Radiation Oncology* Biology* Physics, 35:721-30.

12. Vlachaki MT, Teslow TN, Amosson C, Uy NW, Ahmad S (2005) IMRT versus conventional 3DCRT on prostate and normal tissue dosimetry using an endorectal balloon for prostate immobilization. Medical Dosimetry, 30(2): 69-75.

13. Huang SH, Catton C, Jezioranski J, Bayley A, Rose S, Rosewall T (2008) The effect of changing technique, dose, and PTV margin on therapeutic ratio during prostate radiotherapy. International Journal of Radiation Oncology* Biology* Physics, 71(4): 1057-64.

14. Pollack A, Zagars GK, Starkschall G, Antolak JA, Lee JJ, et al. (2002) Prostate cancer radiation dose response: results of the MD Anderson phase III randomized trial. International Journal of Radiation Oncology* Biology* Physics, 53(5): 1097-1105.
15. D'Amico AV, Manola J, Loffredo M, Renshaw AA, DellaCroce A, Kantoff PW (2004) 6-month androgen suppression plus radiation therapy vs radiation therapy alone for patients with clinically localized prostate cancer: a randomized controlled trial. JAMA, 292(7): 821-827.

16. Rimmer $\mathrm{Y}$, Burnet $\mathrm{N}$, Routsis $\mathrm{D}$, Twyman $\mathrm{N}$, Hoole $\mathrm{A}$, et al. (2008) Practical issues in the implementation of imageguided radiotherapy for the treatment of prostate cancer within a UK department. Clinical Oncology, 20(1): 22-30.

17. Freedman GM, Anderson PR, Li J, Eisenberg DF, Hanlon AL, et al. (2006) Intensity modulated radiation therapy (IMRT) decreases acute skin toxicity for women receiving radiation for breast cancer. American Journal of Clinical Oncology, 29(1): 66-70.

18. Ashman JB, Zelefsky MJ, Hunt MS, Leibel SA, Fuks Z (2005) Whole pelvic radiotherapy for prostate cancer using $3 D$ conformal and intensity-modulated radiotherapy. International Journal of Radiation Oncology* Biology* Physics, 63 (3): 765-771.

19. Vanhavere F, Huyskens D, Struelens L (2004) Peripheral neutron and gamma doses in radiotherapy with an $18 \mathrm{MV}$ linear accelerator. Radiation protection dosimetry, 110(14): 607-612.

20. Podgorsak E, Rawlinson J, Johns H (1975) X-ray depth doses from linear accelerators in the energy range from 10 to $32 \mathrm{MeV}$. American Journal of Roentgenology, 123(1): 182-191.

21. Palma D, Vollans E, James $K$, Nakano S, Moiseenko V, et al. (20080 Volumetric modulated arc therapy for delivery of prostate radiotherapy: comparison with intensitymodulated radiotherapy and three-dimensional conformal radiotherapy. International Journal of Radiation Oncology* Biology* Physics, 72(4): 996-1001.

22. Martin J, Frantzis J, Eade T, Chung P (2010) Clinician's guide to prostate IMRT plan assessment and optimisation. Journal of Medical Imaging and Radiation Oncology, 54(6): 569-575. 
